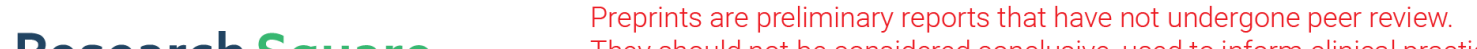 They should not be considered conclusive, used to inform clinical practice, or referenced by the media as validated information. \\ Transverse Kerker Effect in All-Dielectric Spheroidal Particles
}

\author{
Mikhail M. Bukharin
}

National University of Science and Technology "MISiS"

Vladimir Ya. Pecherkin

Joint Institute for High Temperatures

Anar K. Ospanova

National University of Science and Technology "MISiS"

Vladimir B. Ilin

St. Petersburg State University

Leonid M. Vasilyak

Joint Institute for High Temperatures

Alexey A. Basharin ( $\square$ Alexey.basharin@gmail.com )

National University of Science and Technology "MISiS"

Boris Luk'yanchuk

Lomonosov Moscow State University

\section{Research Article}

Keywords: spheroidal, Kerker effect, lasers

Posted Date: October 1st, 2021

DOl: https://doi.org/10.21203/rs.3.rs-947900/v1

License: (1) (1) This work is licensed under a Creative Commons Attribution 4.0 International License.

Read Full License 


\title{
Transverse Kerker effect in all-dielectric spheroidal particles
}

\author{
Mikhail M. Bukharin ${ }^{1}$, Vladimir Ya. Pecherkin ${ }^{2}$, Anar K. Ospanova ${ }^{1,3}$, Vladimir B. Il'in ${ }^{4,5,6}$, \\ Leonid M. Vasilyak ${ }^{2}$, Alexey A. Basharin ${ }^{1,7, *}$, and Boris Luk'yanchuk ${ }^{8}$
}

${ }^{1}$ National University of Science and Technology "MISiS", The Laboratory of Superconducting Metamaterials and
Department of Theoretical Physics and Quantum Technologies, Moscow, 119049, Russia
2Joint Institute for High Temperatures, Russian Academy of Sciences, Moscow, 125412, Russia
${ }^{3}$ al-Farabi Kazakh National University, Department of Physics and Technology, Almaty, 050040, Kazakhstan
${ }^{4}$ St. Petersburg State University, Dept. Math. Mechan., St. Petersburg, 198504, Russia
${ }^{5}$ Petersburg University of Aerospace Instrumentation, St. Petersburg, 190000, Russia
${ }^{6}$ Main (Pulkovo) Astronomical Observatory of RAS, St. Petersburg, 196140, Russia
${ }^{7}$ Institute for Theoretical and Applied Electromagnetics RAS, Moscow 125412, Russia
${ }^{8}$ Lomonosov Moscow State University, Faculty of Physics, Moscow, 119991, Russia
${ }^{*}$ Alexey.basharin@gmail.com

ABSTRACT

Kerker effect is one of the unique phenomena in modern electrodynamics. Due to overlapping of electric and magnetic dipole moments, all-dielectric particles can be invisible in forward or backward directions. In our paper we propose new conditions between resonantly excited electric dipole and magnetic quadrupole in ceramic high index spheroidal particle for demonstrating transverse Kerker effect. Moreover, we perform proof-of-concept microwave experiment and demonstrate dumbbell radiation pattern with suppressed scattering in both forward and backward direction and enhanced scattering in lateral direction. Our concept is promising for future planar lasers, nonreflected metasurface and laterally excited waveguides and nanoantennas.

\section{Introduction}

Recently, the study of light manipulation in electromagnetic structures have emerged as promising scientific fields due to unusual multipoles interactions in subwavelength all-dielectric and plasmonic particles ${ }^{1}$. The transition from electronic systems and information processing methods to optical ones strongly requires development of high Q-factor open resonators and nanoantennas enabling effective light controlling at the nanoscale in optical microcircuits ${ }^{2}$. However, an implementation of strongly resonance nanoparticles requires complex geometries at nanoscale and forces to search for qualitatively new solutions ${ }^{3}$.

In this issue, all-dielectric nanophotonics brings the idea of resonant scattering on dielectric nanoparticles based on the so-called Mie resonances ${ }^{4}$. Firstly introduced for spherical particles, its definition was extended to particles of various shapes and based on electromagnetic multipoles interactions. There is a plenty of research on dielectric particles of different geometries demonstrating strong fields localizations, Fano-resonances ${ }^{5}$, Kerker conditions ${ }^{6}$, anapole modes ${ }^{7}$, invisible metasurfaces ${ }^{8}$ and Bound states in the continuum (BIC) ${ }^{9}$.

Primarily, invisible physics inspired us to study multipolar interference between multipoles of so-called trivial and nontrivial families ${ }^{6}$. For instance first and second Kerker effects are results of interaction between electric and magnetic dipole moments fulfilling condition for zero backward or forward scattering ${ }^{10,11}$. Similarly, Kerker effect can be observed in interaction between quadrupoles of electric and magnetic types ${ }^{6}$. For the first time, Kerker effect was experimentally demonstrated for ceramic sphere in microwave ${ }^{12}$ and then for silicon nanosphere ${ }^{13}$ and gallium arsenide nano-disks in optics ${ }^{14}$. The main problem for demonstrating Kerker effects in all-dielectric particles is overlapping between resonances of electric and magnetic dipole moments in the same frequency ${ }^{15}$. For example, the magnetic dipole and electric dipole resonances are separated from each other for silicon nanosphere. The solution can be found in the application of spheroidal particles giving optimum aspect ratio for overlapping electric and magnetic resonances provide suppressed backward scattering and strong forward scattering ${ }^{16}$.

The second level of Kerker-scattering can be defined as suppression of radiation in both forward and backward directions and named as generalized Kerker effect $t^{6,17}$. In this issue, the particles of disks, cuboids shape ${ }^{18}$ and onions multilayer particles ${ }^{17}$ can be considered invisible due to interactions between electric and magnetic dipoles as well as between their quadrupoles. Moreover, metasurfaces based on them are almost transparent and unaccompanied by phase change with light transmission ${ }^{8}$. From practical applications, simultaneous excitation of Kerker's first and second conditions becomes crucially important for 
strong near-field localization and developing of nonreflected metasurfaces for advanced photonic technologies.

For the first glance, generalized Kerker effect may seem similar to electric and magnetic anapole states and hybrid anapole as well ${ }^{19-21}$. However, the main difference is that generalized Kerker effect accompanied by transverse scattering while anapole particle does not scatter at all exceptionally up to higher multipoles in agreement with optical theorem ${ }^{22-26}$. On the other hand, the side scattering property can be usefully exploited for metasurfaces as a platform for planar lasers via transverse Kerker effect as coupling between near fields of particles and for lateral excitation of nano-waveguides ${ }^{2,8,18}$.

For this aim, we theoretically propose and perform proof-of-concept microwave experiment of intensive lateral scattering by spheroidal all-dielectric particle. We demonstrate this effect on the spheroidal particle with ratio close to 2.1 between coincided resonantly electric dipole and magnetic quadrupole moments which gives transverse radiation pattern of dumbbell form in the selected direction perpendicular to the incident wave front.

We note that the study of electromagnetic response of spheroidal particles is significant by several reasons. Some natural space systems are very close to spheroidal shapes since dust grains in the interplanetary and interstellar medium are assumed to have spheroidal shape ${ }^{27,28}$ and their response may be explained by our approach. Moreover, spheroidal particles are equally important for the problem of medicine and microbiology ${ }^{29}$.

Additionally, resonant scattering phenomena have been demonstrated in another nonspherical particles. Nonradiating mode conditioned by so-called hybrid anapole mode establishment demonstrated in high-index dielectric ellipsoidal particle ${ }^{21}$ and in all-dielectric nanocylinders ${ }^{30}$.

\section{Dumbbell radiation due to multipoles interaction}

We start with finding of condition for transverse scattering from the multipole decomposition of the field radiated by the arbitrary particle ${ }^{31}$. We note, that transverse Kerker effect is interpreted as suppression of both scattering in forward and backward directions providing enhanced scattering in lateral directions ${ }^{18}$.

In our consideration, we use only families of dipoles and quadrupoles.

The radiation of arbitrary source is formulated by electric field of multipoles. For $l=1$, radiation is presented by electric and magnetic dipoles, while for $l=2$ by electric and magnetic quadrupoles:

$$
\begin{aligned}
& \mathbf{E}_{(t o t a l)}(\boldsymbol{\theta}, \boldsymbol{\varphi}, r) \approx \mathbf{E}_{(l=1)}+\mathbf{E}_{(l=2)}, \\
& \mathbf{E}_{(l=1)}(\boldsymbol{\theta}, \varphi, r) \approx \frac{\mu_{0} c^{2}}{3 \sqrt{2 \pi}} \frac{\exp (-i k r)}{r} \sum_{m=0, \pm 1}\left[\left(k^{2} Q_{1, m}-i k^{3} T_{1, m}\right) \times\left(\mathbf{Y}_{1,2, m}+\sqrt{2} \mathbf{Y}_{1,0, m}\right)+i \sqrt{3}\left(k^{2} M_{1, m} \times \mathbf{Y}_{1,1, m}\right)\right], \\
& \mathbf{E}_{(l=2)}(\boldsymbol{\theta}, \varphi, r) \approx \frac{\mu_{0} c^{2}}{10 \sqrt{6 \pi}} \frac{\exp (-i k r)}{r} \sum_{m=0, \pm 1, \pm 2}\left[i k^{3} Q_{2, m}^{(e)} \times\left(\sqrt{2} \mathbf{Y}_{2,3, m}+\sqrt{3} \mathbf{Y}_{2,1, m}\right)-\sqrt{5} k^{3} Q_{2, m}^{(m)} \times \mathbf{Y}_{2,2, m}\right] .
\end{aligned}
$$

Here, $\mu_{0}$ is the magnetic permeability of vacuum, $c$ is the speed of light, $r$ is the radius-vector, and $\boldsymbol{Y}_{l, l, m}$ are the spherical vector harmonics. The spherical multipoles are related to the Cartesian multipoles, that is, electric dipole $\boldsymbol{p}$, magnetic dipole $\boldsymbol{M}$, toroidal dipole moment $\boldsymbol{T}$, electric quadrupole $Q e$, magnetic quadrupole $Q m$, as follows:

$$
\begin{aligned}
& Q_{1,0}=p_{z}, \quad Q_{1, \pm 1}=\frac{\mp p_{x}+i p_{y}}{\sqrt{2}}, \quad T_{1,0}=T_{z}, \quad T_{1, \pm 1}=\frac{\mp T_{x}+i T_{y}}{\sqrt{2}}, \quad M_{1,0}=-M_{z}, \quad M_{1, \pm 1}=\frac{ \pm M_{x}-i M_{y}}{\sqrt{2}}, \\
& Q e_{2,0}=3 Q e_{z z}, \quad Q e_{2, \pm 1}=\sqrt{6}\left(\mp Q e_{x z}+i Q e_{y z}\right), \quad Q e_{2, \pm 2}=\frac{\sqrt{6}}{2}\left(Q e_{x x} \mp i 2 Q e_{x y}-Q e_{y y}\right), \\
& Q m_{2,0}=-\frac{3}{2} Q m_{z z}, \quad Q m_{2, \pm 1}=\sqrt{\frac{3}{2}}\left( \pm Q m_{x z}-i Q m_{y z}\right), \quad Q m_{2, \pm 2}=\frac{\sqrt{6}}{4}\left(-Q m_{x x} \pm i 2 Q m_{x y}+Q m_{y y}\right) .
\end{aligned}
$$

Cartesian multipoles are calculated by integrating over the charge current density $\boldsymbol{J}(\boldsymbol{r})$ distribution within the particle volume and $\alpha, \beta, \gamma=x, y, z$ :

$$
p_{\alpha}=\frac{1}{i \omega} \int J_{\alpha} d^{3} r, \quad m_{\alpha}=\frac{1}{2 c} \int[r \times J]_{\alpha} d^{3} r, \quad T_{\alpha}=\frac{1}{10 c} \int\left[(r \cdot J) r_{\alpha}-2 r^{2} J_{\alpha}\right] d^{3} r, \quad P_{\alpha}=-p_{\alpha}+i k T_{\alpha},
$$




$$
Q e_{\alpha \beta}=\frac{1}{i 2 \omega} \int\left[r_{\beta} J_{\alpha}+r_{\alpha} J_{\beta}-\frac{2}{3}(r \cdot J) \delta_{\alpha \beta}\right] d^{3} r, \quad Q m_{\alpha \beta}=\frac{1}{3 c} \int\left[r_{\alpha}[r \times J]_{\beta}+r_{\beta}[r \times J]_{\alpha}\right] d^{3} r .
$$

Here $\delta_{\alpha \beta}$ is the Kronecker symbol.

The scattering cross section of the particle can be described by terms of multipoles:

$$
\sigma_{\text {scat }}=\frac{k^{4}}{6 \pi \varepsilon_{0}^{2}\left|E_{0}\right|}\left(|M|^{2}+|P|^{2}\right)+\frac{k^{6}}{80 \pi \varepsilon_{0}^{2}\left|E_{0}\right|}\left(4\left|Q e_{\alpha \beta}\right|^{2}+\left|Q m_{\alpha \beta}\right|^{2}\right) .
$$

Let us suppose two transverse scattering cases, mutually perpendicular to each other:

A. $\theta=0$ and $\theta=\pi$. In this case multipoles radiate only in transverse to incident wave direction and scattering fields $\boldsymbol{E}_{(\text {total })}(\theta=0)=0$ and $\boldsymbol{E}_{(\text {total })}(\theta=\pi)=0$.

B. $\theta=\pi / 2$ and $\theta=3 \pi / 2$. In this case multipoles radiate only along incident wave direction and scattering fields $\boldsymbol{E}_{(\text {total })}(\theta=\pi / 2)=0$ and $\boldsymbol{E}_{(\text {total })}(\theta=3 \pi / 2)=0$.

From equation (1) one can simply find solution for conditions 1 and 2 (see Methods) and we get relations between electric dipole $\boldsymbol{P}$ and magnetic quadrupole $Q_{m}$. However, we imply other multipoles have insufficient response in our system:

$$
\begin{aligned}
& P_{\alpha}=-\frac{i k}{2} Q m_{\beta \gamma}, \\
& P_{\alpha}=\frac{i k}{2} Q m_{\beta \gamma} .
\end{aligned}
$$

The same conditions can be obtained for magnetic dipole and electric quadrupole.

This simple result reveals that zero radiation (Transverse Kerker effect) simultaneously in backward and forward directions (Condition 1, equation 3) or simultaneously in lateral directions (Condition 2, equation 4) can be achievable just by interference between two multipoles of electric dipole moment and magnetic quadrupole. Moreover, the radiation direction almost defined by phase of magnetic quadrupole which indicated by its sign. Similar results were obtained numerically by Asano at al..$^{32}$, but without explaining the reason for side scattering.

Recently, Jeng Yi Lee et al. ${ }^{17}$ formulated conditions for simultaneously nearly zero forward and backward scattering. It is possible when the first (dipoles) and second (quadrupoles) order multipoles excited in particle are obeyed the condition $a_{1}=-5 / 3 b_{2}$ and $b_{1}=-5 / 3 a_{2}$. However, for simple all-dielectric sphere the coexistence of electric and magnetic dipoles and their quadrupoles is impossible at the same frequencies. Then, the multilayer structures ${ }^{17}$, spherical particles with radial anisotropy give chance for experimental evidence of transverse Kerker effect ${ }^{33}$.

To realize this unique property, let us consider the electromagnetic scattering by high-index dielectric spheroidal particle with $a$ and $b$ being the major and minor semiaxis, respectively. This ratio between $a / b$ indicates the shape of spheroid form changing from needle $(a / b>1$, prolate spheroid), passing by sphere $(a / b=1)$, to disk $(a / b<1$, oblate spheroid). During our experimental study, we suppose the case of prolate spheroid with $a=19.5 / 2 \mathrm{~mm}$ and $b=12.5 / 2 \mathrm{~mm}$, and ratio $a / b=1.56$. The dielectric permittivity of particle is $\varepsilon=150$ that presented as high-index ceramics. The particle is illuminated by plane wave at lateral incidence with polarization of vector $\boldsymbol{E}$ parallel to minor spheroidal axis $x$. The particle geometry and excited wave are depicted on Fig. 1.

In our paper, we demonstrate spheroidal all dielectric particle as promising for demonstration of transverse Kerker effect due to extra radius as a channel for multipoles tunability in comparison with sphere.

For this, we consider the spheroid with shape given by equation

$$
\frac{x^{2}}{b^{2}}+\frac{y^{2}}{a^{2}}+\frac{z^{2}}{b^{2}}=1 .
$$

In order to demonstrate transverse scattering approach, we perform simulation of electromagnetic scattering by spheroidal particle for different ratio between $a / b$ (Fig. 2). We use commercial version of CST Microwave Studio and Time domain solver with open boundary conditions. The particle is illuminated by a plane wave with linearly polarized $\boldsymbol{E}$ component (Figure 1). The scattering cross section normalized to $\sigma / \pi a b$. For small ratio $a / b<1$, the particle is of disk shape and its scattering spectrum is defined by two main resonances where magnetic dipole moment $M$ is the firsts and electric dipole $P$ is the second term. This trend is not changing up to $a / b=1$, i.e. transition disk to the sphere. We observe that the bifurcation point $a / b=1$ is split into two 
resonances for $a / b>1$. Both of them are characterized by electric dipole moment and magnetic quadrupole moment. However, the main difference between them is determined in the phases of the magnetic quadrupoles. For the first case (Transverse scattering), electric dipole moment $P_{x}$ component and magnetic quadrupole $Q m_{y z}$ have positive sign of phases, while the second resonance (Longitudinal scattering) is characterized by the opposite sign of phases.

To propose features of electromagnetic response of prolate spheroid $(a / b>1)$ and their modes, we consider the spectra of particle with $\mathrm{a} / b=1.56$ and demonstrate its experimental scattering cross-section (Fig. 3). The resonance peeks on $1.8 \mathrm{GHz}, 2.17$ $\mathrm{GHz}$ and $2.65 \mathrm{GHz}$ demonstrate good agreement between theory and experiment.

In order to clearly understand the origin of peaks, we perform multipole decomposition of four main multipoles excited in system, where $\boldsymbol{P}$ means electric dipoles, $\boldsymbol{M}$ magnetic ones, $Q e$ electric quadrupoles and $Q m$ magnetic ones for first and second resonances. The first peak close to $1.8 \mathrm{GHz}$ is almost defined by magnetic response $\boldsymbol{M}$ (Fig. 4). Other multipoles are sufficiently suppressed and do not contribute to the response of the system. We also recalculated the shape of the scattering resonance from multipoles, which in good agreement with CST calculated scattering cross section.

The second resonance at $2.17 \mathrm{GHz}$ is a result of interaction between components of electric dipole $P_{x}$ and magnetic quadrupole $Q m_{y z}=Q m_{z y}$ (Fig. 5). Other multipoles tend to zero and do not contribute to the response of the system.

The third peak at $2.65 \mathrm{GHz}$ is also characterized by electric dipole $P_{x}$ and magnetic quadroupole $Q m_{y z}$ components (Fig. 6). Indeed, scattering responses at $2.17 \mathrm{GHz}$ (Fig. 5a) and $2.65 \mathrm{GHz}$ (Fig. 6a) have identical multipoles contributions. However, we analyze their phases close to resonances in order to find difference between them. The phases on $2.175 \mathrm{GHz}$ (Fig. 5b) of electric and magnetic quadrupole are both positive and $1.752 \mathrm{rad}$ and $0.1338 \mathrm{rad}$, respectively. However, the phase of magnetic quadrupole is negative on $2.648 \mathrm{GHz}$ and $-1.9 \mathrm{rad}$ (Fig. 6b). In order to demonstrate resulted radiation pattern of resonance peak $2.175 \mathrm{GHz}$ and $2.648 \mathrm{GHz}$, we plot radiation patterns of electric dipole and magnetic quadrupole taken into account their amplitudes and phases. For resonance at $2.175 \mathrm{GHz}$ (Fig. 5c), electric dipole and magnetic quadrupole radiate with positive phases, accordingly radiation of their interference enhanced in $y$ direction and suppressed along $z$ axis. Contrary, radiation on $2.648 \mathrm{GHz}$ (Fig. 6c) formed by destructive interference of electric dipole and magnetic quadrupole in $y$ direction, but radiation in $z$ direction is identical in forward and backward directions.

Thus, the scattering pattern of all-dielectric spheroidal particle is defined by electric and magnetic quadrupole and its phase sign. Our simulation results in good agreement with equation (3).

\section{Proof-of-concept experiment}

In order to observe transverse scattering we perform proof-of-concept experiment and measured radiation pattern at $2.17 \mathrm{GHz}$ in two planes $x y$ and $y z$. For this purpose, we fabricate ceramic spheroidal particle with large axis of $2 a=19.5 \pm 0.1 \mathrm{~mm}$ and small axis $2 b=12.5 \pm 0.1 \mathrm{~mm}$ made of K15U-2 capacitor ceramic. The value of the dielectric permittivity is varied in the range $\varepsilon=140-170$ with frequencies with a loss tangent $\tan \delta=10^{-4}-10^{-3}$.

We use home-made anechoic chamber for measurement of scattering cross sections and radiation patterns of all-dielectric spheroidal particle. The distance between the wideband horn antenna ETS-Lindgren's model 3115 and the spheroid is 45 $\mathrm{cm}$. For registration of transmission and reflection coefficients we exploit Agilent E5071C ENA Network Analyzer. We also use $20 \mathrm{~dB}$ gain amplifier to increase the signal-to-noise ratio. For each test, the background radiation level was additionally measured in the presence of incident microwave radiation without the ceramic particle. Our setup permits to measure signal variation on the scale up to $-80 \mathrm{~dB}$.

Obviously, the normalized radiation patterns in both planes of spheroid remind the dumbbell shape and coincide with the simulated pattern (Fig. 7) and we observe strong transverse scattering, reminiscent patterns resulted as interference of electric dipole and magnetic quadrupole multipoles (3) while forward and backward scattering is negligible. We also add theoretical pattern in manner of formula 3 for comparison with theoretical results. Due to our experiment we observe suppression scattering in 0 and 180 grads directions. However, the scattering in lateral 90 and 270 grads directions is pronounced.

Recently, the authors actively discuss the transverse Kerker effect of suppression scattering in forward or backward direction. However, Ufimtsev introduced another principle of invisibility ${ }^{34}$. He shown in 1962 (English translated in 1971) that the waves can bend around of body and diffracted in lateral directions. He called this waves as edges waves. Moreover, our effect of dumbbell-form scattering is different from invisibility effect due to enhanced scattering cross-section, while Kerker effect and Ufimtsev scattering aim to reduce scattering in whole.

In summary, we would like to remark that implementation of such dumbbell scattering with near zero forward/scattering can be fabricated without complicated anisotropic and multilayer systems, as well as without strong loss plasmonic materials. For a spherical particle, it is impossible to simultaneously suppress forward and backward scattering due to the optical theorem. However, for spheroids and other geometries, the optical theorem is modified to provide suppression of both forward/backward scattering in the presence of strong side scattering.Moreover, we propose that in optical experiments spheroidal particles may be replaced by elliptical cylinders. The long-awaited problem in nanophotonics of planar lasers excited laterally would be 
realized by metasurfaces of proposed particles. Note, that transverse dumbbell Kerker effect can be realized in planar structures by interaction of magnetic dipoles and electric quadrupoles.

\section{Conclusion}

In this work, we proposed conditions for transverse dumbbell scattering based on interaction between multipoles of electric dipole and magnetic quadrupole moments. Our condition is beyond invisibility effects like classical and transverse Kerker effect and also anapole mode with suppressed scattering cross-section. In contrary, our scattering is enhanced in lateral directions. Therefore, by fabricated all-dielectric ceramic spheroidal particle based on K15U-2 capacitor ceramic, we proposed proof-of-concept microwave experiment and demonstrated dumbbell scattering in perpendicular to incident wave. At the same time, we observed suppressed forward and backward scattering. Our concept can be applied as ingredients for lateral excitation of planar lasers, waveguides and nanoantennas.

\section{Methods}

The radiation of arbitrary source is formulated by electric field of multipoles. For $l=1$, radiation is presented by electric and magnetic dipoles, while for $l=2$ by electric and magnetic quadrupoles. Due to electric and toroidal dipoles radiate with the same radiation pattern in far-field zone, we combine them as total electric dipole $\boldsymbol{P}$

$$
\begin{aligned}
& \mathbf{E}_{(P)} \approx \frac{\mu_{0} c^{2}}{3 \sqrt{2 \pi}} \frac{\exp (-i k r)}{r} \sum_{m=0, \pm 1}\left[\left(k^{2} Q_{1, m}-i k^{3} T_{1, m}\right) \times\left(\mathbf{Y}_{1,2, m}+\sqrt{2} \mathbf{Y}_{1,0, m}\right)\right], \\
& \mathbf{E}_{(Q m)} \approx \frac{\mu_{0} c^{2}}{10 \sqrt{6 \pi}} \frac{\exp (-i k r)}{r} \sum_{m=0, \pm 1, \pm 2}\left[-\sqrt{5} k^{3} Q_{2, m}^{(m)} \times \mathbf{Y}_{2,2, m}\right] \\
& \mathbf{E}_{(\text {total })} \approx \mathbf{E}_{(P)}+\mathbf{E}_{(Q m)} .
\end{aligned}
$$

For $\varphi=\frac{\pi}{2}$ and $\widehat{x}, \widehat{y}, \widehat{z}$-basis in Cartesian coordinates

$$
\begin{aligned}
E(\theta, \varphi=\pi / 2) \approx & \left(C_{1} P_{x}+\frac{C_{2}}{4}\left(4 Q m_{y z} \cos 2 \theta+\left(Q m_{y y}-Q m_{x x}-3 Q m_{z z}\right) \sin 2 \theta\right)\right) \widehat{x} \\
& +\left(C_{1}\left(P_{y} \cos \theta-P_{z} \sin \theta\right) \cos \theta-C_{2}\left(Q m_{x z} \cos \theta+Q m_{x y} \sin \theta\right) \cos \theta\right) \widehat{y} \\
& +\left(C_{1}\left(P_{z} \sin \theta-P_{y} \cos \theta\right) \sin \theta+C_{2}\left(Q m_{x z} \cos \theta+Q m_{x y} \sin \theta\right) \sin \theta\right) \widehat{z} .
\end{aligned}
$$

Here $C_{1}$ and $C_{2}$ are $\theta$-independent constants:

$$
C_{1}=\frac{e^{-i k r} c^{2} \mu_{0} k^{2}}{4 \pi r}, \quad C_{2}=\frac{i e^{-i k r} c^{2} \mu_{0} k^{3}}{8 \pi r} .
$$

We can obtain condition for dumbbell radiation pattern in $\widehat{x}, \widehat{y}, \widehat{z}$ directions or along $\boldsymbol{E}, \boldsymbol{H}, \boldsymbol{k}$.

It is following from equation (6) for $\theta=0$ and $\theta=\pi$ (transverse scattering) or $\theta=\pi / 2$ for and $\theta=3 \pi / 2$ for simultaneous suppressed forward/backward scattering

$$
E_{(\text {total })}(\theta, \phi=\pi / 2, r=1) \approx C_{1} P_{\alpha}+C_{2} Q m_{\beta \gamma} \cos 2 \theta=0
$$

We note, that components $Q m_{x x}, Q m_{y y}, Q m_{z z}$ radiate with zero $\boldsymbol{E}$-fields for transverse scattering due to $\sin 2 \theta=0$. However, function $\cos 2 \theta$ for other components of magnetic quadrupole forms four-lobe radiation pattern.

Accordingly, we obtain 3 conditions for dumbbell radiation pattern along $\boldsymbol{E}, \boldsymbol{H}, \boldsymbol{k}$ vectors:

1. For radiation pattern along $\boldsymbol{H}$ vector and suppressed scattering along $\boldsymbol{k}$ and $\boldsymbol{E}$, as in our experiment:

$$
C_{1} P_{x}+C_{2} Q m_{y z}=0, \quad C_{1} P_{y}-C_{2} Q m_{x z}=0 .
$$

For multipoles, we have

$$
\frac{P_{x}}{Q m_{y z}}=-\frac{i k}{2}, \quad \frac{P_{y}}{Q m_{x z}}=\frac{i k}{2} .
$$


2. For radiation pattern along $\boldsymbol{k}$ vector and suppressed scattering along $\boldsymbol{E}$ and $\boldsymbol{H}$ :

$$
C_{1} P_{x}-C_{2} Q m_{y z}=0, \quad C_{1} P_{z}+C_{2} Q m_{x y}=0 .
$$

For multipoles:

$$
\frac{P_{x}}{Q m_{y z}}=\frac{i k}{2}, \quad \frac{P_{z}}{Q m_{x y}}=-\frac{i k}{2} .
$$

3. For radiation pattern along $\boldsymbol{E}$ vector and suppressed scattering along $\boldsymbol{k}$ and $\boldsymbol{H}, \varphi=0$ :

$$
C_{1} P_{y}+C_{2} Q m_{x z}=0, \quad C_{1} P_{z}-C_{2} Q m_{x y}=0 .
$$

For multipoles:

$$
\frac{P_{y}}{Q m_{x z}}=-\frac{i k}{2}, \quad \frac{P_{z}}{Q m_{x y}}=\frac{i k}{2} .
$$

\section{References}

1. Grahn, P., Shevchenko, A. \& Kaivola, M. Electromagnetic multipole theory for optical nanomaterials. New J. Phys. 14, 093033 (2012).

2. Novotny, L. \& Hecht, B. Principles of Nano-Optics (Cambridge Univ. Press, Cambridge, 2012).

3. Kuznetsov, A. I., Miroshnichenko, A. E., Brongersma, M. L., Kivshar, Y. S. \& Luk'yanchuk, B. S. Optically resonant dielectric nanostructures. Science 354, 6314 (2016).

4. Jackson, J. Classical Electrodynamics (Wiley, NY, 1975).

5. Luk'yanchuk, B. S. et al. The Fano resonance in plasmonic nanostructures and metamaterials. Nat. Mater. 9, 707-715 (2010).

6. Liu, W. \& Kivshar, Y. S. Generalized Kerker effects in nanophotonics and meta-optics [Invited]. Opt. Expr. 26, 13085-13105 (2018).

7. Miroshnichenko, A. E. et al. Nonradiating anapole modes in dielectric nanoparticles. Nat. Comm. 6, 8069 (2015).

8. Shamkhi, H. K. et al. Transparency and perfect absorption of all-dielectric resonant metasurfaces governed by the transverse Kerker effect. Phys. Rev. Mater. 3, 085201 (2019).

9. Koshelev, K., Lepeshov, S., Liu, M., Bogdanov, A. \& Kivshar, Y. Asymmetric metasurfaces with high-Q resonances governed by bound states in the continuum. Phys. Rev. Lett. 121, 193903 (2018).

10. Kerker, M., Wang, D. \& Giles, G. Electromagnetic scattering by magnetic spheres. J. Opt. Soc. Am. 73, $765-767$ (1983).

11. Evlyukhin, A. B. et al. Demonstration of magnetic dipole resonances of dielectric nanospheres in the visible region. Nano Lett. 12, 3749-3755 (2012).

12. Geffrin, J. M. et al. Magnetic and electric coherence in forward- and back-scattered electromagnetic waves by a single dielectric subwavelength sphere. Nat. Commun. 3, 1171 (2012).

13. Fu, Y. H., Kuznetsov, A. I., Miroshnichenko, A. E., Yu, Y. F. \& Luk'yanchuk, B. S. Directional visible light scattering by silicon nanoparticles. Nat. Commun. 4, 1527 (2013).

14. Person, S. et al. Demonstration of zero optical backscattering from single nanoparticles. Nano Lett. 13, 1806-1809 (2013).

15. Staude, I. et al. Tailoring directional scattering through magnetic and electric resonances in subwavelength silicon nanodisks. ACS Nano 7, 7824-7832 (2013).

16. Luk'yanchuk, B. S., Voshchinnikov, N. V., Paniagua-Domínguez, R. \& Kuznetsov, A. I. Optimum forward light scattering by spherical and spheroidal dielectric nanoparticles with high refractive index. ACS Photon. 2, 993-999 (2015).

17. Lee, J. Y., Miroshnichenko, A. E. \& Lee, R.-K. Simultaneously nearly zero forward and nearly zero backward scattering objects. Opt. Expr. 26, 30393-30399 (2018).

18. Shamkhi, H. K. et al. Transverse scattering and generalized Kerker effects in all-dielectric Mie-resonant metaoptics. Phys. Rev. Lett. 122, 193905 (2019). 
19. Zanganeh, E. et al. Anapole meta-atoms: nonradiating electric and magnetic sources. Phys. Rev. Lett. 127, 096804 (2021).

20. Luk'yanchuk, B., Paniagua-Domínguez, R., Kuznetsov, A. I., Miroshnichenko, A. E. \& Kivshar, Y. S. Hybrid anapole modes of high-index dielectric nanoparticles. Phys. Rev. A 95, 063820 (2017).

21. Ospanova, A. K., Basharin, A. A., Miroshnichenko, A. E. \& Luk'yanchuk, B. S. Generalized hybrid anapole modes in all-dielectric ellipsoid particles. Opt. Mater. Express 11, 23 (2021).

22. Luk'yanchuk, B., Paniagua-Domínguez, R., Kuznetsov, A. I., Miroshnichenko, A. E. \& Kivshar Y. S. Suppression of scattering for small dielectric particles: anapole mode and invisibility. Phil. Trans. R. Soc. A 375, 20160069 (2017).

23. Nemkov, N. A., Basharin, A. A. \& Fedotov, V. A. Nonradiating sources, dynamic anapole, and Aharonov-Bohm effect. Phys. Rev. B 95, 165134 (2017).

24. Nemkov, N. A., Basharin, A. A. \& Fedotov, V. A. Electromagnetic sources beyond common multipoles. Phys. Rev. A 98, 023858 (2018).

25. Papasimakis, N., Fedotov, V. A., Savinov, V., Raybould, T. A. \& Zheludev, N. I. Electromagnetic toroidal excitations in matter and free space. Nat. Mater. 15, 263-271 (2016).

26. Gurvitz, E. A. et al. The high-order toroidal moments and anapole states in all-dielectric photonics. Laser Photon. Rev. 13, 1800266 (2019).

27. Freeman, K. C. The galactic spheroid and old disk. Ann. Rev. Astron. Astrophys. 25, 603-632 (1987).

28. Bahcall, J. N., Schmidt, M. \& Soneira, R. M. The galactic spheroid. Astrophys. J. 265, 730-747 (1983).

29. Fennema, E., Rivron, N., Rouwkema, J., Blitterswijk, C. \& Boer, J. Spheroid culture as a tool for creating 3D complex tissues. Trends Biotechn. 31, 108-115 (2013).

30. Valero A. C. et al. Theory, observation, and ultrafast response of the hybrid anapole regime in light scattering. Laser Photon. Rev. 15, 2100114 (2021).

31. Savinov, V., Fedotov, V.A. \& Zheludev, N. I. Toroidal dipolar excitation and macroscopic electromagnetic properties of metamaterials. Phys. Rev. B 89, 205112 (2014).

32. Asano, S. \& Yamamoto, G. Light scattering by a spheroidal particle. Appl. Opt. 14, 29-49 (1975).

33. Ni, Y. X., Gao, L., Miroshnichenko, A. E. \& Qiu, C. W. Controlling light scattering and polarization by spherical particles with radial anisotropy. Opt. Expr. 21, 8091-8100 (2013).

34. Ufimtsev, P. Method of Edge Waves in the Physical Theory of Diffraction (Dayton, U.S. Air Force Foreign Technology Division, 1971).

\section{Acknowledgements}

This work was supported by Ministry of Science and Higher Education of the Russian Federation (grant 14.W03.31.0008). This work was partially supported by the Russian Science Foundation (No. 20-12-00389). The multipole decomposition calculations was supported by Russian Science Foundation (No. 20-72-00016). AAB work of analytical study of electromagnetic scattering was funded by the Russian Science Foundation (No. 21-19-00138) The reported study was funded by RFBR, project number 20-02-00715. The experimental study of measuring spectra and the magnetic intensity distribution was supported by the Ministry of Science and Higher Education of the Russian Federation (State Assignment No. 075-00460-21-00). V.I. was partly supported by the SUAI subsidy FSRF-2020-0004 and the grants RFBR 18-52-52006, RSF 20-72-10052.

\section{Author contributions statement}

M.M.B., A.K.O., V.B.I., A.A.B. and B.L. designed and performed the numerical simulation, multipole decomposition of the structure. V.Ya.P., L.M.V fabricated sambles and performed microwave experiments. All authors discussed the results and approved the manuscript. All authors reviewed the manuscript.

\section{Additional information}

Competing interests. The authors declare no competing interests.

Correspondence and requests for materials should be addressed to A.A.B. 
(a)

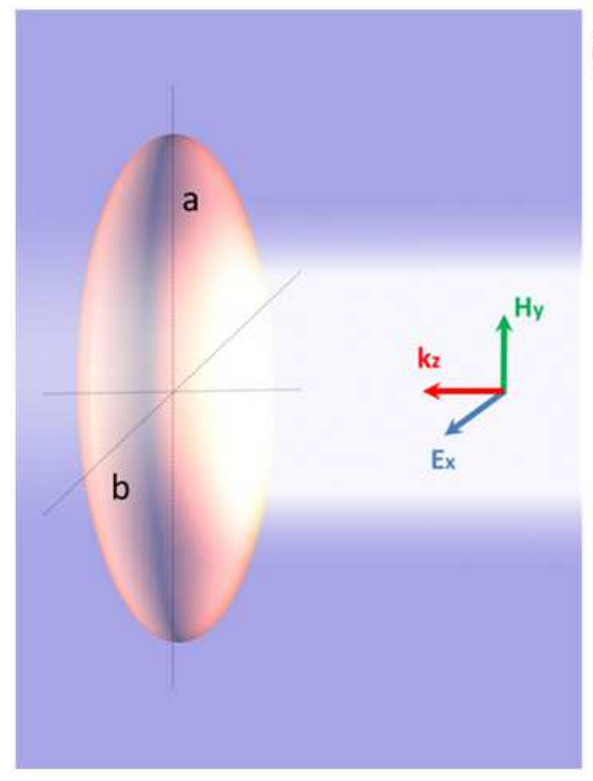

(b)

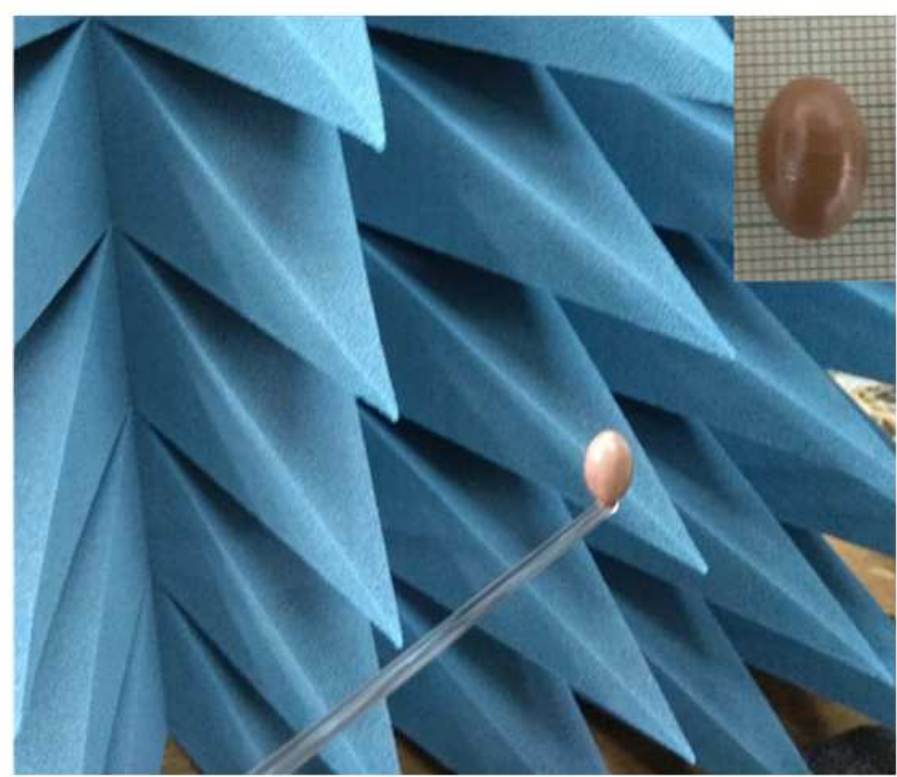

Figure 1. (a) Illustration of high-index dielectric prolate spheroidal particle with dielectric permittivity $\varepsilon=150$ and major and minor semiaxes of $a$ and $b$, respectively. Linearly polarized plane wave is impinging laterally with vector $\boldsymbol{E}$ parallel to minor axis, (b) experimental sample of ceramics spheroidal particle with $a=19.5 / 2 \mathrm{~mm}$ and $b=12.5 / 2 \mathrm{~mm}$ in anechoic chamber. The inset shows picture of sample.

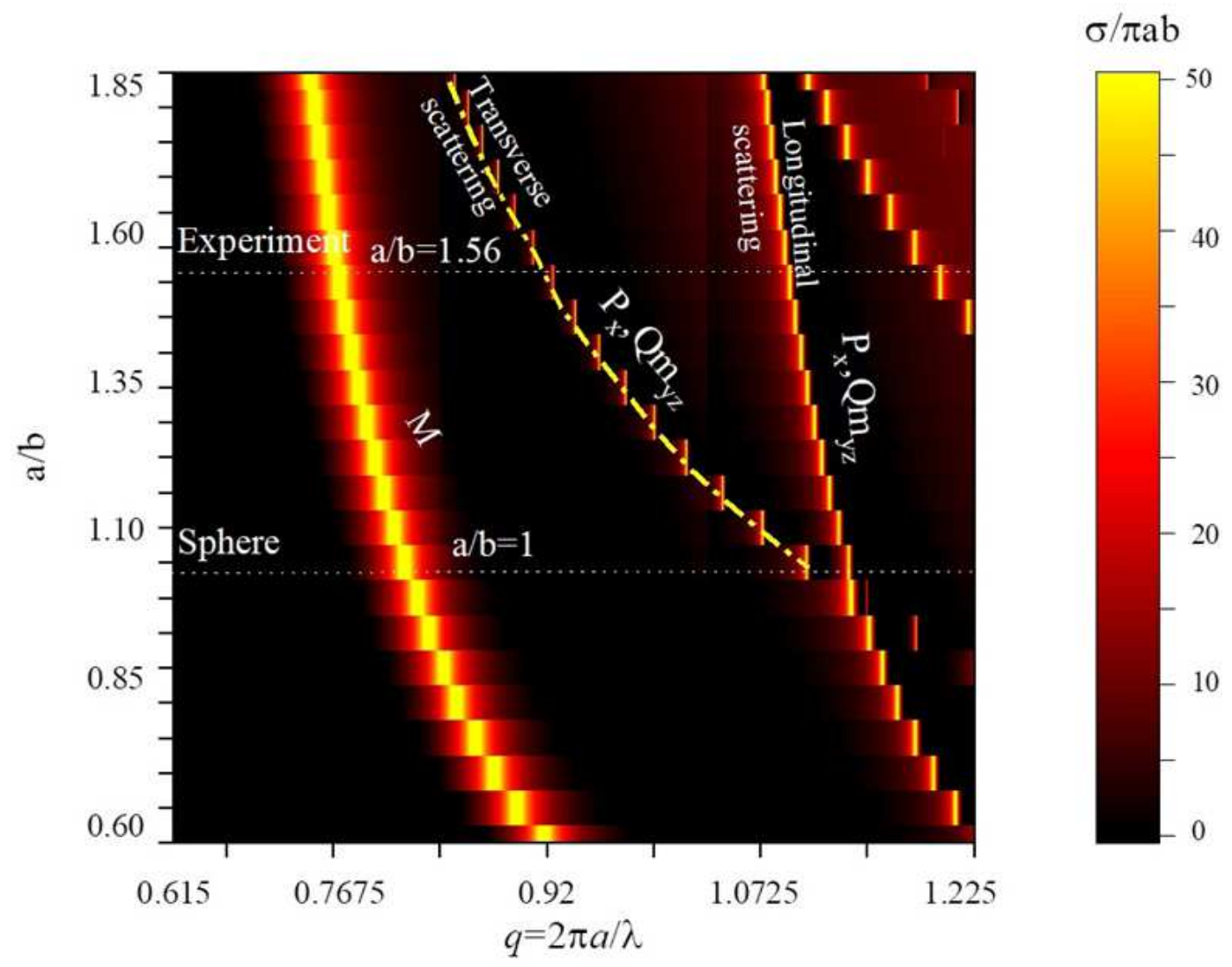

Figure 2. Scattering cross-section of spheroidal all-dielectric particle depending on the aspect ratio of the semiaxes $a / b$. 


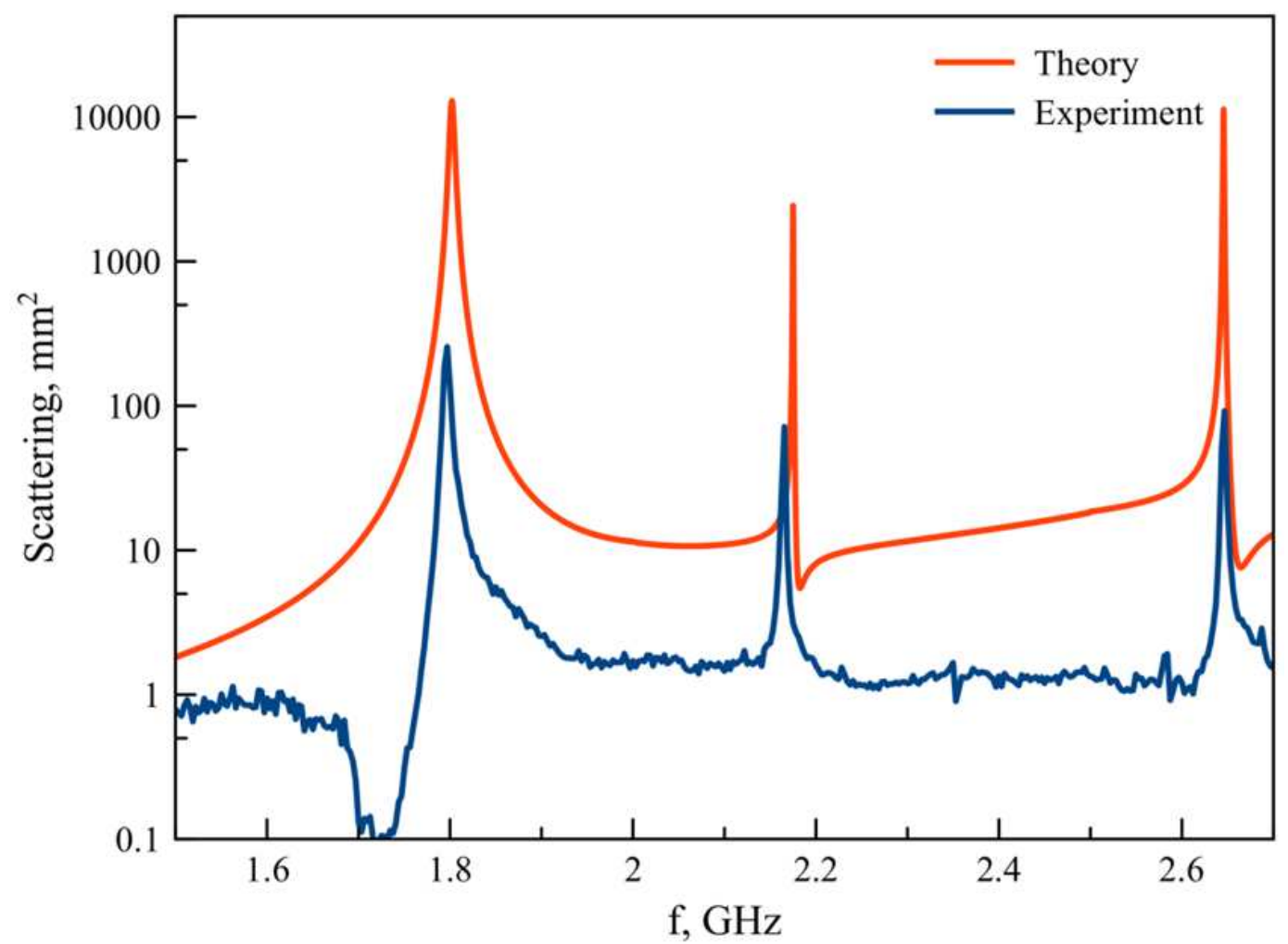

Figure 3. Simulated and measured scattering characteristics of spheroid with aspect ratio $a / b=1.56$ at $1.5-2.5 \mathrm{GHz}$ frequency range.

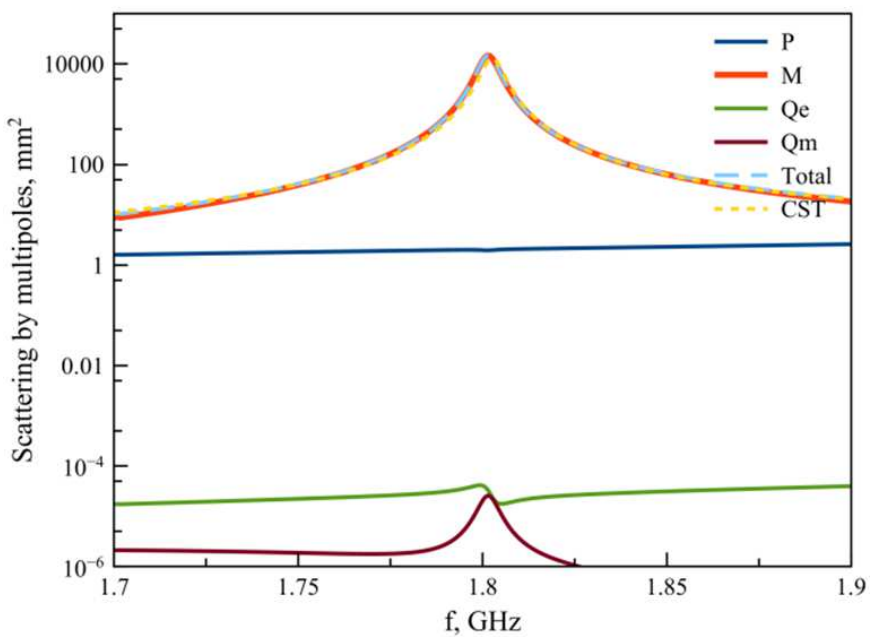

Figure 4. Scattering cross section of multipoles on $1.8 \mathrm{GHz}$. 
(a)

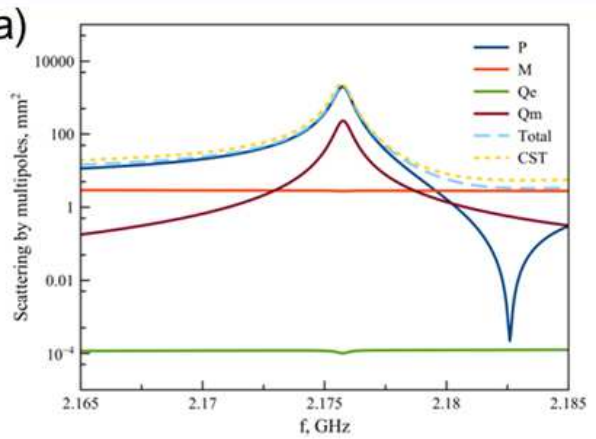

(c)

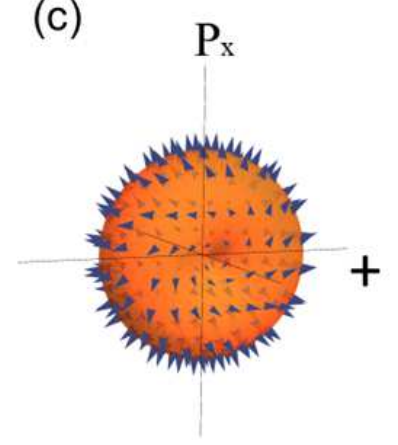

(b)

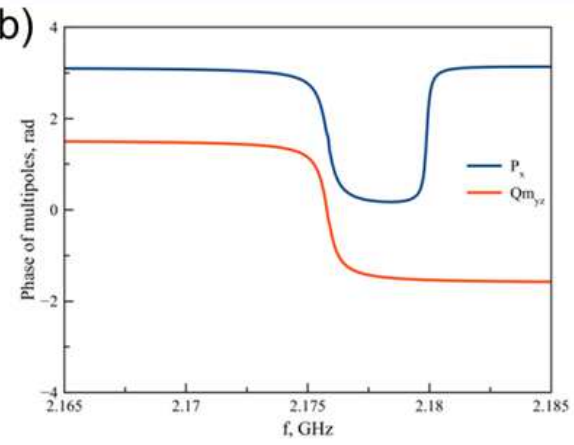

Transverse scattering

Figure 5. (a) Scattering cross section of multipoles on $2.175 \mathrm{GHz}$. (b) Phases of electric dipole and magnetic quadrupole. (c) Radiation pattern of excited electric dipole and magnetic quadrupole and total radiation pattern.

(a)

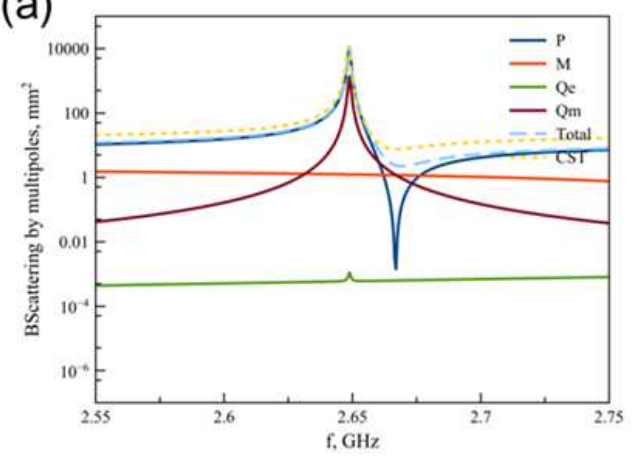

(c)

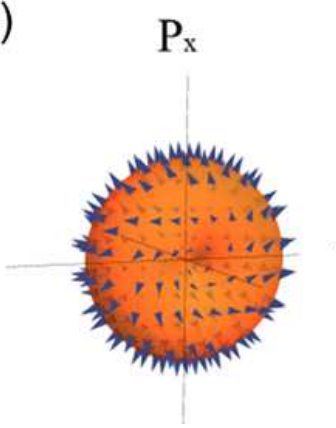

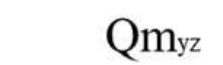

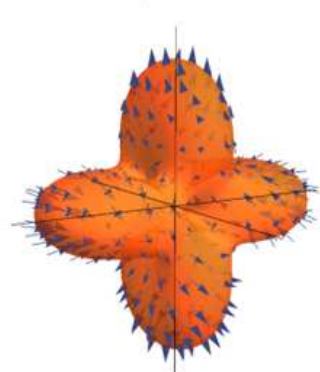

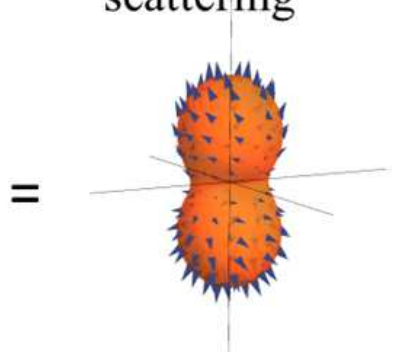

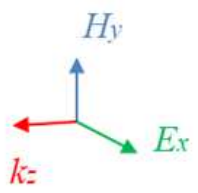

$k=$

(b)

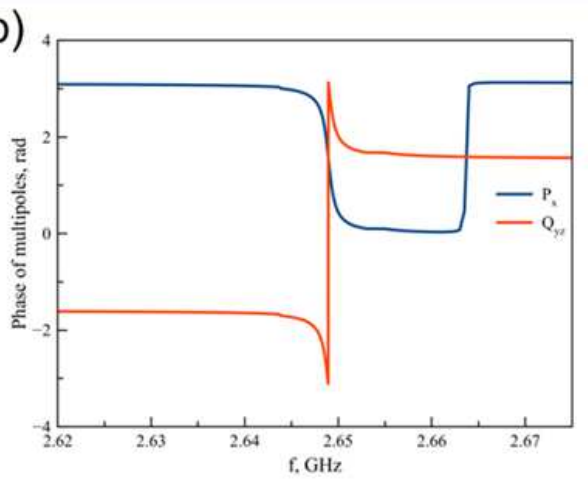

Forward/backward scattering
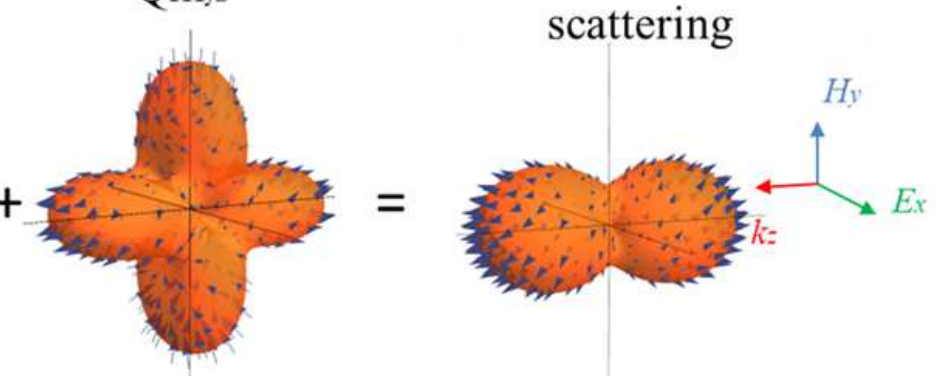

Figure 6. (a) Scattering cross section of multipoles on $2.64 \mathrm{GHz}$. (b) Phases of electric dipole and magnetic quadrupole. (c) Radiation pattern of excited electric dipole and magnetic quadrupole and total radiation pattern. 


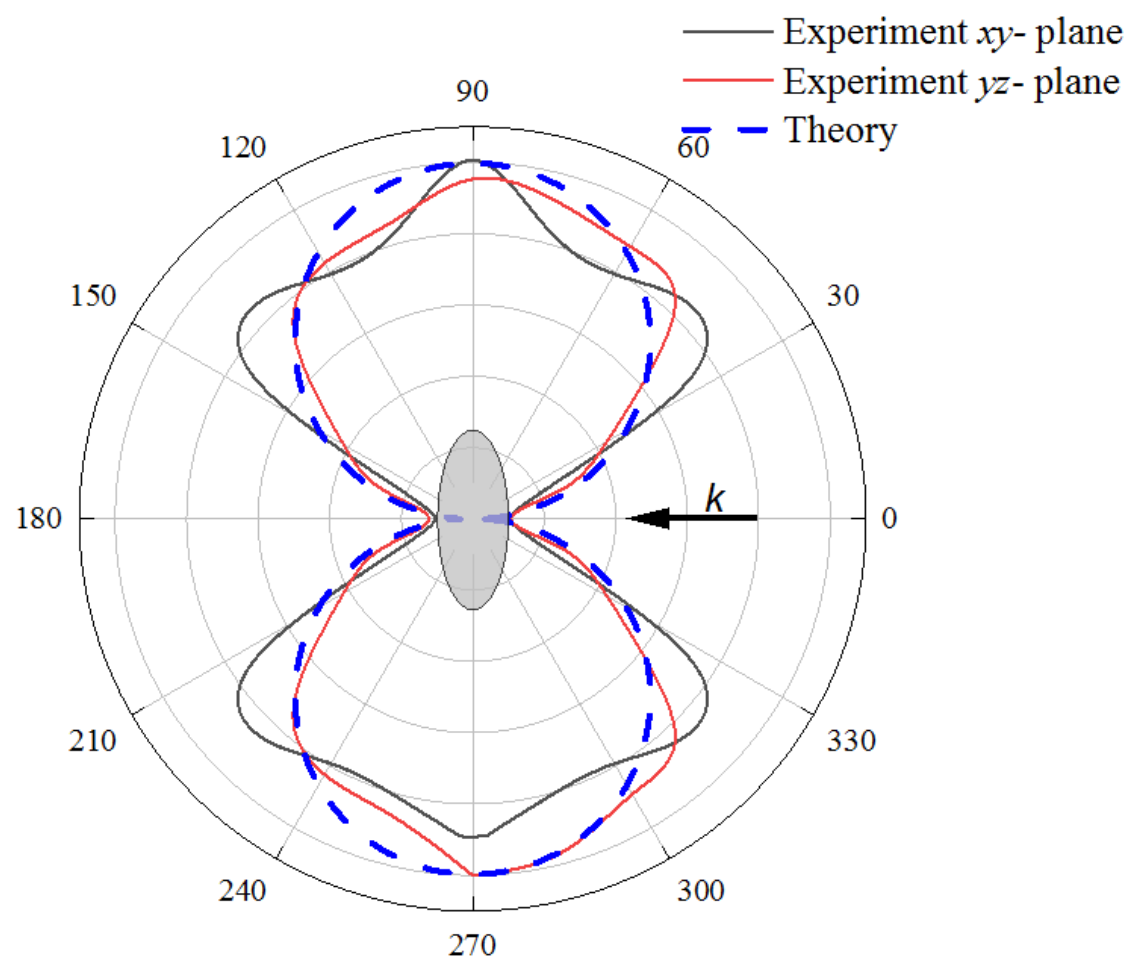

Figure 7. Experimental radiation pattern on $2.17 \mathrm{GHz}$ for $x y$-plane, $y z$-plane compared with theory. 